\title{
Studying Voltage Stability in Power System Considering Load Dynamics
}

\author{
H. Radmanesh' ${ }^{1 *}$, S. S. Heidari Yazdi², S. U. Mosazadeh² and G. B. Gharehpetian² \\ 'Department of Electrical Engineering, Islamic Azad University, Takestan Branch, Takestan, Iran; \\ Hamid.radmanesh@aut.ac.ir \\ 2Electrical Engineering Department, Amirkabir University of Technology, Tehran, Iran; \\ saedheidary@aut.ac.ir, s_yosuf_mosazade@yahoo.com, grptian@aut.ac.ir
}

\begin{abstract}
This paper investigates on-load tap changer OLTC effect on voltage stability. Chaos theory used in this analysis is via the calculation of the maximum power transmission. Resulted chaotic behavior is exhibited by singular induced bifurcation which clearly shows the voltage stability of the power system with OLTC from a singular induced bifurcation point of view. The paper is organized as follows: 1) increasing in the maximum reactive power via increasing the ratio of OLTC and the possibility of voltage collapse decrease under the same load command. 2) Noting that the voltage instability is directly affected by the load characteristics, shown via the effect of OLTC on the voltage instability under different static load models. 3) Studying voltage stability in state space and the effects of the generator excitation current limit, the OLTC and load dynamics on voltage collapse. In addition, a small-disturbance voltage stability region of a power system is constructed. The voltage stability of the system is determined by the dynamic characteristics of both the OLTC and the load.
\end{abstract}

Keywords: On Load Tap Changer, Voltage Stability, State Space, Bifurcation Diagram.

\section{Introduction}

In the last three decades, worldwide, voltage collapse system black-outs have stimulated researchers' interest on voltage stability [1,2]. The on-load tap changer (OLTC) has a significant influence on voltage stability [3, 4]. The limitation of maximum power transfer by OLTC has been discussed [5]. A technique for on-line automatic condition assessment of OLTC via a self-organizing map has been reported [6]. The control sequence for improving the controllers include transformers, generators, and shunt capacitors [7]. The vibration and fault detection of power systems with OLTC via the wavelet theory has been studied $[8,9]$. The dynamic mechanism of the voltage collapse due to the OLTC operation has been presented [10], where the voltage collapse process has been reconstructed employing a nonlinear dynamic model of the OLTC, the static impedance load and decoupled reactive power-voltage equations. The dynamic process of the voltage collapse has been analyzed based on load, OLTC dynamics and using the load bus voltage $V$ as a state variable in the load model $[11,12]$. Dynamic and static aspects of voltage collapse have been qualitatively investigated [13] and has presented a time-domain simulation to analyze the effects of the OLTC and load dynamics on the voltage collapse in the input/output parameter space $(P, V)[14]$. It has shown that the action of the OLTC plays an important role in the voltage collapse procedure. When the dynamics of the OLTC and the load arc have been separately considered, there are only active power recovery in the dynamic load according to the law of the conservation of energy and voltage recovery at the secondary side of the OLTC. This paper studies the effect of load and OLTC characteristics, the generator excitation current limit, OLTC and load dynamics on the voltage stability.

${ }^{*}$ Corresponding author:

H. Radmanesh (Hamid.nsa@gmail.com) 


\section{Dynamic Model of Power Systems Considering OLTC and Nonlinear Load}

The analyzed power system consists of a Synchronous generator, a transmission line, an OLTC and a nonlinear static load as shown in Figure 1.

Suppose that the OLTC is lossless, the system can be simplified as shown in Figure 2.

The dynamic equation of the generator can be written as

$$
\dot{E}_{q}^{\prime}=-\frac{x_{d}}{T_{d 0}^{\prime} x_{d}^{\prime}} E_{q}^{\prime}+\frac{x_{d}-x_{d}^{\prime}}{T_{d 0} x_{d}^{\prime}} V_{G} \cos \left(\delta_{G}-\delta\right)+\frac{1}{T_{d 0}^{\prime}} E_{f a}
$$

The regulation dynamic of the excitation system can be expressed by the following equation:

$$
\dot{E}_{f d}=\frac{1}{T}\left(-E_{f d}+E_{e d 0}-K\left(V_{G}-V_{r e f}\right)\right)
$$

The power flow equation of the system is as follows:

$$
\begin{aligned}
& 0=\frac{E^{\prime}{ }^{\prime} V_{G}}{x^{\prime} d} \sin \left(\theta_{G}-\delta\right)+\frac{n V_{G} V}{X} \sin \left(\theta_{G}-\theta\right)
\end{aligned}
$$

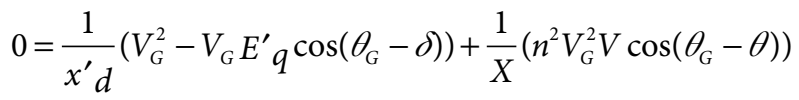

where,

$$
0=\frac{1}{X} n V_{G} V \sin \left(\theta-\theta_{G}\right)+P
$$

and

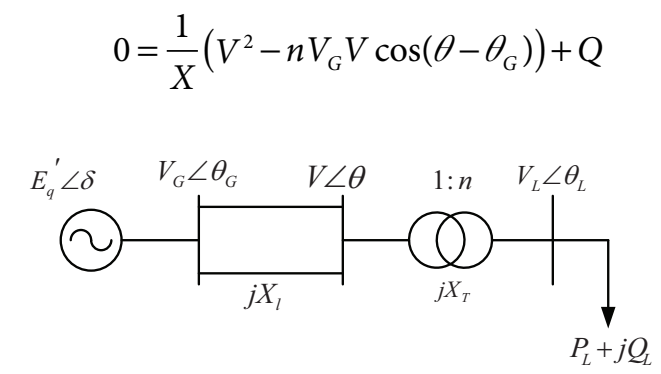

Figure 1. Power system including OLTC and Nonlinear Load.

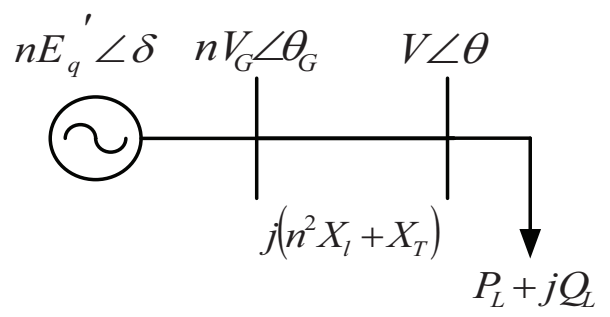

Figure 2. Simplified model of power system. where, $\delta$ is the power angle of the generator, $E_{q}^{\prime}$ is the $\mathrm{q}$-axis internal transient voltage of the generator, $E_{\mathrm{fd}}$ is the voltage of the field circuit of the generator and $V_{\mathrm{G}}$ is the voltage of the generator terminal bus, $X_{d}^{\prime}$ is the $\mathrm{d}$-axis transient reactance of the generator, $X_{\mathrm{d}}$ is the $\mathrm{d}$-axis reactance and $T^{\prime}{ }_{\mathrm{d} 0}$ is the $\mathrm{d}$-axis transient open-circuit time constant.

Using (5) and (6), the following equation can be written:

$$
V_{G}^{2}=\frac{1}{n^{2} V^{2}}\left((X P)^{2}+\left(X Q+V^{2}\right)^{2}\right)
$$

The equation (3) and (4) can be simplified as follows:

$$
0=\frac{1}{X^{\prime}} n E_{q}^{\prime} V \sin \left(\theta-\theta_{G}\right)+P
$$

and

$$
0=\frac{1}{X^{\prime}}\left(V^{2}-n E^{\prime}{ }_{q} V \cos (\theta-\delta)\right)+Q
$$

So, we have:

$$
E_{q}^{\prime 2}=\frac{1}{n^{2} V^{2}}\left(\left(X^{\prime} P\right)^{2}+\left(X^{\prime} Q+V^{2}\right)^{2}\right)
$$

Hence:

$$
\begin{aligned}
& E^{\prime}{ }_{q} V_{G} \cos \left(\theta_{G}-\delta\right)=\frac{1}{n^{2} X V^{2}}\left(X+n^{2} x^{\prime} d\right) \\
& \left((X P)^{2}+\left(X Q+V^{2}\right)^{2}\right)-\frac{x_{d}^{\prime}\left(X Q+V^{2}\right)}{X}
\end{aligned}
$$

where, $X^{\prime}=X+n^{2} X_{\mathrm{d}}^{\prime}, X=n^{2} X_{\mathrm{L}}+X_{\mathrm{T}}, X_{\mathrm{L}}$ is the reactance of the transmission line and $X_{\mathrm{T}}$ is the reactance of the transformers. According to equations (7)-(11), the dynamic of the power system can be written as follows:

$$
\dot{E}_{q}^{\prime}=\frac{1}{T^{\prime} d 0}
$$

$$
\left.\begin{array}{l}
\left\{\begin{array}{l}
-\frac{x_{d}}{x^{\prime} d} E^{\prime} q+\frac{x_{d}-x^{\prime} d}{x^{\prime} E^{\prime} q} \\
{\left[\begin{array}{l}
\frac{1}{n^{2} X V^{2}} \\
\left(X+n^{2} x^{\prime} d\right)\left(\begin{array}{l}
(X P)^{2}+ \\
\left(X Q+V^{2}\right)^{2}
\end{array}\right)
\end{array}\right.} \\
-\frac{x^{\prime} d\left(X Q+V^{2}\right)}{X}
\end{array}\right]+E_{f d} \\
\dot{E}_{f d}=\frac{1}{T}\left[\begin{array}{l}
-K\left(\frac{\sqrt{(X P)^{2}+\left(X Q+V^{2}\right)^{2}}}{n V}\right. \\
\left.-V_{r e f}\right)-E_{f d}+E_{f d 0}
\end{array}\right.
\end{array}\right\}
$$




$$
\begin{aligned}
& 0=n^{2} E_{q}^{2} V^{2}-\left(X^{\prime} P\right)^{2}-\left(X^{\prime} P\right)^{2}- \\
& \left(X^{\prime} Q+V^{2}\right)^{2}
\end{aligned}
$$

\section{Effect of OLTC on System Active and Reactive Powers}

The equation (14) can be written as follows:

$$
\left(\frac{n V_{G} V}{X}\right)^{2}=P^{2}+\left(Q+\frac{V^{2}}{X}\right)^{2}
$$

or,

$$
Q=\sqrt{\left(\frac{n V_{G} V}{X}\right)^{2}-P^{2}}-\frac{V^{2}}{X}
$$

Now the curves $Q$ versus $V$ can be plotted as shown in Figure 3 and Figure 4 for $n=1$ and $n=3$, respectively.

As shown in Figures 3 and 4, the maximum reactive power increases when the ratio of OLTC increases too. So, the possibility of the voltage collapse will decrease. Rewriting the equation (16) in the following form:

$$
\left(\frac{V^{2}}{X}\right)^{2}+\left(2 Q-\frac{n^{2} V_{G}^{2}}{X}\right)\left(\frac{V^{2}}{X}\right)+\left(P^{2}+Q^{2}\right)=0
$$

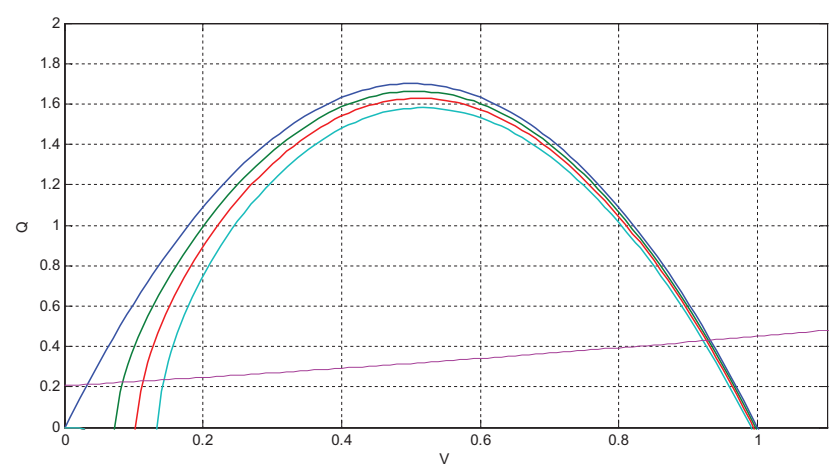

Figure 3. Q-V curve for $\mathrm{n}=1$.

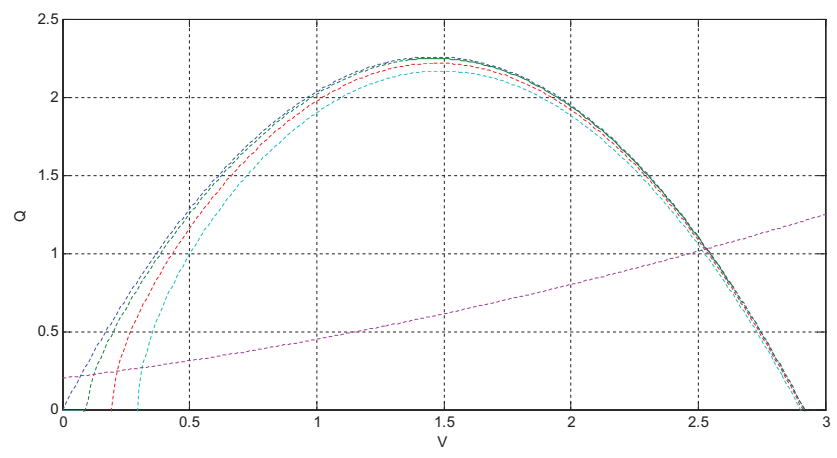

Figure 4. $\mathrm{Q}-\mathrm{V}$ curve for $\mathrm{n}=3$.
The P-Q equation can be written as follows:

$$
\left(2 Q-\frac{n^{2} V_{G}^{2}}{X}\right)^{2}-4\left(P^{2}+Q^{2}\right)=0
$$

The variation of $P$ versus $Q$ is shown in Figure 5 .

$$
Q=\frac{n^{2} V_{G}^{2}}{4 X}-\frac{X P^{2}}{n^{2} V_{G}^{2}}
$$

Now, the maximum of $Q$ can be determined, as follows:

$$
Q_{\max }=\frac{n^{2} V_{G}^{2}}{4 X}=\frac{n^{2} V_{G}^{2}}{4\left(n^{2} X_{L}+X_{T}\right)}
$$

From equation(16), we have:

$$
P \leq \frac{V \sqrt{n^{2} V_{G}^{2}-V^{2}}}{X}=\frac{V \sqrt{n^{2} V_{G}^{2}-V^{2}}}{n^{2} X_{L}+X_{T}}
$$

So, the maximum active power decreases along with increasing of $n$.

\section{Stability Analysis Based on Chaos Theory}

In this section, the condition of the singular induced bifurcation is discussed and the effect of the OLTC tap ratio of OLTC on the voltage stability is studied. First, it is assumed that the power factor of the load is constant i.e., $P=\eta Q$. Considering the equation (20) the following algebraic equation can be written:

$$
0=n^{2} E_{q}^{\prime 2} V^{2}-\left(X^{\prime} \eta Q\right)^{2}-\left(X^{\prime} Q+V^{2}\right)^{2}
$$

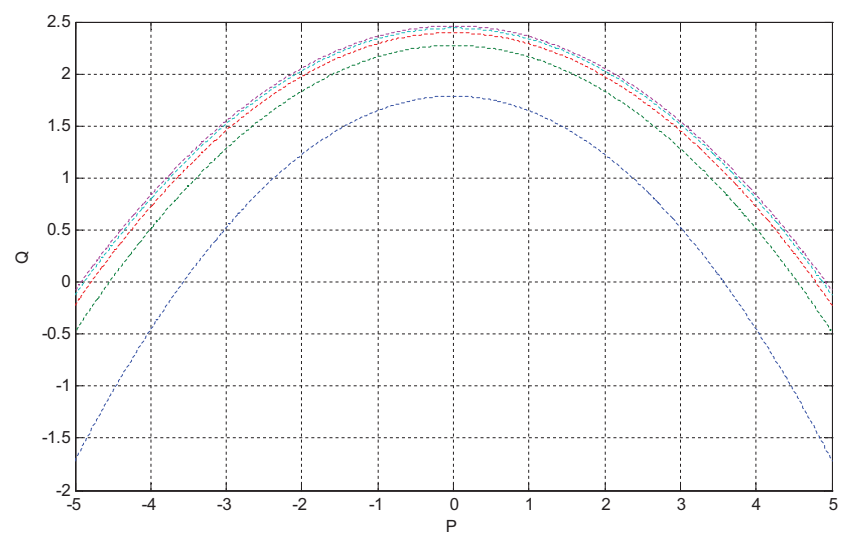

Figure 5. Q-P curve for different tap ratios. 
Let

$$
g\left(E_{q}^{\prime}, V\right)=n^{2} E_{q}^{\prime 2} V^{2}-\left(X^{\prime} \eta Q\right)^{2}-\left(X^{\prime} Q+V^{2}\right)^{2}
$$

If there exist a singular induced bifurcation, then we have $\frac{\partial g}{\partial V}=0$, i.e., $n^{2} E_{q}^{\prime 2}=2\left(X^{\prime} Q+V^{2}\right)$ and we have:

$$
\begin{aligned}
& V_{S}^{2}=X^{\prime} \sqrt{P^{2}+Q^{2}}=X^{\prime} S=\left(n^{2} X_{L}+X_{T}+n^{2} x_{d}^{\prime}\right) S \\
& E_{q}^{\prime 2}=\frac{1}{n^{2}} X^{\prime}(Q+S)=\frac{n^{2} X_{L}+X_{T}+n^{2} x_{d}^{\prime}}{n^{2}}(Q+S)
\end{aligned}
$$

For the power systems, if $V^{2} \geq X^{\prime} S$ or, $E_{q}^{\prime 2} \geq \frac{1}{n^{2}} X^{\prime}(Q+S)$, the power system can be run safely [4]. Using this inequality, the effect of the OLTC on the possibility of the singular induced bifurcation can be discussed as follows:

(1) For the load bus, the higher tap ratio will increase the critical value of the singular induced bifurcation. The system should operate at a higher voltage, then possibility of the singular induced bifurcation will increase for the same load condition.

(2) For the internal transient voltage of the generator, we have $\frac{d E_{q}^{\prime 2}}{d n}=-\frac{2 X_{T}(Q+S)}{n^{3}} \leq 0$, i.e., along with the increasing of the OLTC tap ratio, the critical voltage of the singular induced bifurcation of the generator internal voltage will decrease and the possibility of the bifurcation will increase.

Based on these discussions, it can be said that under same power conditions, increasing of the tap ratio will causes higher possibility of occuring singular induced bifurcation and thus has a negative effect on the voltage stability.

\section{Effect of OLTC on Voltage Instability for Different Load behaviors}

In this section, the effect of OLTC on the voltage instability is studied based on the maximum power and singular induced bifurcation for different load behaviors.

\subsection{Constant Power Load}

The constant power load is modeled by the equations, $P=P_{0}$ and $Q=Q_{0}$. Considering the equation (24) we have:

$$
\begin{aligned}
& 0=V^{4}+2\left(X^{\prime} Q-n^{2} E_{q}^{\prime 2}\right) V^{2}+X^{\prime 2}\left(P^{2}+Q^{2}\right)= \\
& V^{4}+2\left(X^{\prime} Q-n^{2} E_{q}^{\prime 2}\right) V^{2}+X^{\prime 2} S^{2}
\end{aligned}
$$

and,

$$
V^{2}=\frac{1}{2}\left(\sqrt{n^{2} E_{q}^{\prime 2}-2 X^{\prime} Q \pm}\right.
$$

Taking into consideration, the higher voltage operating condition, we have:

$$
V^{2}=\frac{1}{2}\left(\begin{array}{l}
n^{2}{E^{\prime 2}}_{q}^{2}-2 X^{\prime} Q \pm \\
\sqrt{\left(2 X^{\prime} Q-n^{2} E_{q}^{\prime 2}\right)^{2}-4 X^{\prime 2} S^{2}}
\end{array}\right)
$$

If the equation has a solution, then the following inequality must be valid:

$$
E_{q}^{\prime}>\frac{2 X^{\prime}(Q+S)}{n^{2}}
$$

or

$$
E_{q}^{\prime}>\frac{2\left(n^{2} X_{L}+X_{T}+n^{2} x_{d}^{\prime}\right)(Q+S)}{n^{2}}
$$

This equation shows that the internal transient voltage will decrease along with increasing the tap ratio. Then, the possibility of the voltage collapse will decrease.

\subsection{Constant Impedance Load}

For constant impedance load, we have $P=G V^{2}$ and $Q=B V^{2}$. So, we have:

$$
0=V^{4}+2\left(X^{\prime} B V^{2}-n^{2} E_{q}^{\prime 2}\right) V^{2}+X^{\prime 2} V^{4}\left(G^{2}+B^{2}\right)
$$

or

$$
0=\left(1+2 X^{\prime} B+X^{\prime} Z^{2}\right) V^{2}-n^{2} E_{q}^{\prime 2}
$$

where, $Z^{2}=G^{2}+B^{2}$. Solving this equation result in:

$$
V=\frac{n E_{q}^{\prime}}{\sqrt{1+2 X^{\prime} B+X^{\prime} Z^{2}}}
$$

So, there is no singular induced bifurcation and the power system will not have a voltage collapse problem due to the singularity of the algebraic equation. For the constant exponential load, we have $Q=b V^{\beta}$ and $p=a V^{\alpha}$. Due to the complexity of the load characteristic, there is no explicit solution for the algebraic equation. Noting that the constant exponential load can be approximately modeled by the following equation:

$$
\left\{\begin{array}{l}
P=P_{0}+L V+G V^{2} \\
Q=Q_{0}+H V+B V^{2}
\end{array}\right.
$$


Then, this case can be transformed to the combination of the problem of the constant power load, constant current load and constant impedance load.

\section{Power System Modelling for State Space}

The studied power system is shown in Figure 6 (a) and its equivalent thevenin model can be seen in Figure 6 (b). It consists of a transmission Line, a generator, a load an OLTC. The generator is supposed to have a constant terminal voltage when the excitation current does not reach its limit.

As a single step in the tap position contributes a minor voltage correction, a continuous model of the OLTC in $[10]$ is adopted as:

$$
\frac{d n}{d t}=\frac{1}{T_{n}}\left(V_{30}-V_{3}\right)
$$

where, $n$ is the turn ratio, $V_{3}$ and $V_{30}$ are the measured and reference secondary voltages, respectively, and $T_{n}$ is the time constant of the OLTC. The dynamic model presented in [15] is used to analyze the voltage stability. The dynamic load model is the induction motor modeled by a shunt impedance, as shown in Figures 6 and 7, where the dynamic resistance $R_{\mathrm{E}}$ is the function of the slip, $R_{\mathrm{S}}+j X_{\mathrm{S}}$ is the static impedance load, and $X_{\sigma}$ is the reactance between the dynamic resistance $R_{E}$ and the load bus. The resistance $R_{\sigma}$ between $R_{E}$ and the load bus is neglected. The effect of the dynamic resistance $\left(R_{E}\right)$ can be approximately described by the following dynamic equation:

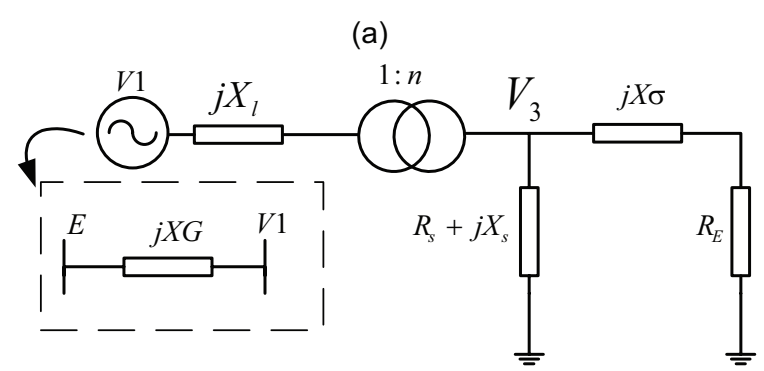

(b)

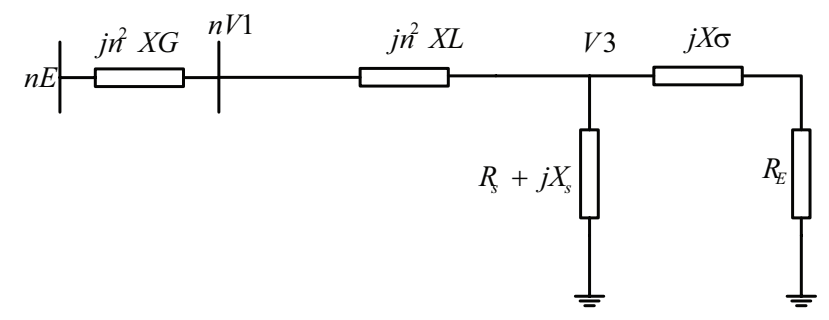

Figure 6. a) Power system one line diagram b) thevenin equivalent model.

$$
\frac{d R_{E}}{d t}=-\frac{R_{E}^{2}}{T_{E}}\left(P_{T}-P_{E}\right)
$$

where, $P_{\mathrm{E}}$ and $P_{\mathrm{T}}$ are the electrical input and mechanical output of the dynamic load, respectively, $T_{\mathrm{E}}$ is the time constant of the dynamic load expressed as $T_{\mathrm{E}}=T_{\mathrm{m}} \cdot R_{\sigma}$, and $T_{\mathrm{m}}$ is the time constant of the aggregate induction motor. Since $R_{\sigma}$ is small, the value of $T_{\mathrm{E}}$ is also small. As shown in Figure 7, when the generator excitation current limit is neglected, the secondary voltage $V_{3}$ of the OLTC is as follows:

$$
V_{3}=\frac{n V_{1} * Z_{3}}{Z_{1}}
$$

where, $Z_{1}=\left|j n^{2} X_{\mathrm{L}}+\left(R_{\mathrm{S}}+j X_{\mathrm{S}}\right) / /\left(R_{\mathrm{E}}+j X_{\sigma}\right)\right|$ and $Z_{3}=\mid\left(R_{\mathrm{S}}+j X_{\mathrm{S}}\right)$ // $\left(R_{\mathrm{E}}+j X_{\sigma}\right) \mid . V_{3}$ can also be written as follows:

$$
V_{3}=\frac{V_{e q} * Z_{E}}{Z_{2}}
$$

where $Z_{1}, Z_{3}, Z_{\mathrm{E}}$, and $Z_{\text {eq }}$ are shown in Figure 7. $V_{\text {eq }}=n V_{1} Z_{\mathrm{S}}$ l $Z_{4}, Z_{\mathrm{E}}=\left|\left(R_{\mathrm{E}}+j X_{\mathrm{o}}\right)\right|, Z_{\mathrm{eq}}=R_{\mathrm{eq}}+\mathrm{j} X_{\mathrm{eq}}=j n^{2} X_{\mathrm{L}} / /\left(R_{\mathrm{S}}+j X_{\mathrm{S}}\right), Z_{2}=\mid R_{\mathrm{eq}}$ $+R_{\mathrm{E}}+\mathrm{j}\left(X_{\mathrm{eq}}+X_{\mathrm{\sigma}}\right) \mid$ and $Z_{4}=\left|R_{\mathrm{S}}+R_{\mathrm{E}}+j\left(n^{2} X_{\mathrm{L}}+X_{\mathrm{S}}\right)\right|$. Considering Figure 7 , the active power absorbed by the dynamic load is also obtained by the following equation:

$$
P_{E}=\frac{n^{2} V_{1}^{2} Z_{3}^{2} R_{E}}{Z_{1}^{2} Z_{E}^{2}}
$$

where, voltage parameters are shown in Figure 8.

Equation (39) can also be written as follows:

$$
P_{E}=\frac{V_{e q}^{2} R_{E}}{Z_{2}^{2}}
$$

\section{OLTC and Load Behavior in Power System}

To study the power system the parameters listed in Table (1), is used.

\subsection{OLTC and Load Analysis}

For $R_{\mathrm{E}}=0.8$, the $V_{3}$ variation versus $n$ is shown in Figure 9 for pre-disturbance and post-disturbance, respectively. Suppose that the initial operating point is at $A^{S}$ on predisturbance curve.

The operating point will jump to point $B$ on curve 2 (postdisturbance) after occurrence of a disturbance, which leads to the reactance change from $X_{\mathrm{L}}^{(1)}$ to $X_{\mathrm{L}}^{(2)}$ with $X_{\mathrm{L}}^{(2)}=0.2$. 


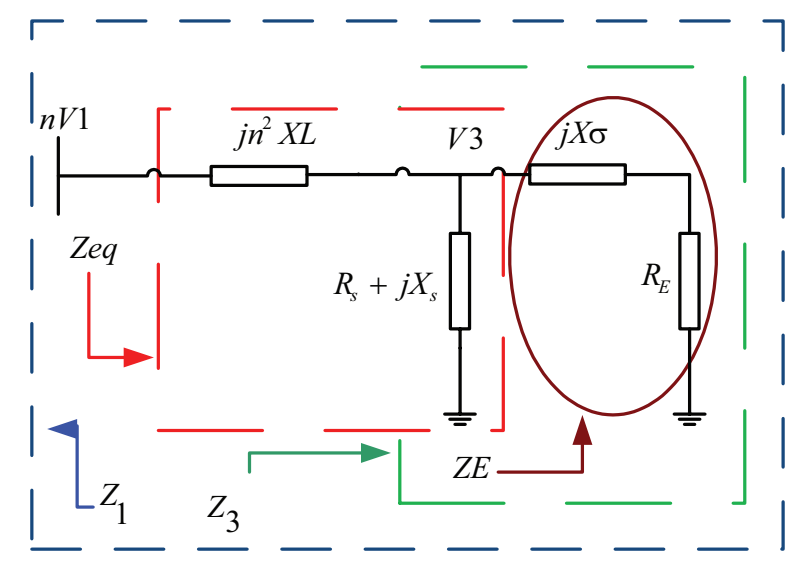

Figure 7. Impedance parameters.

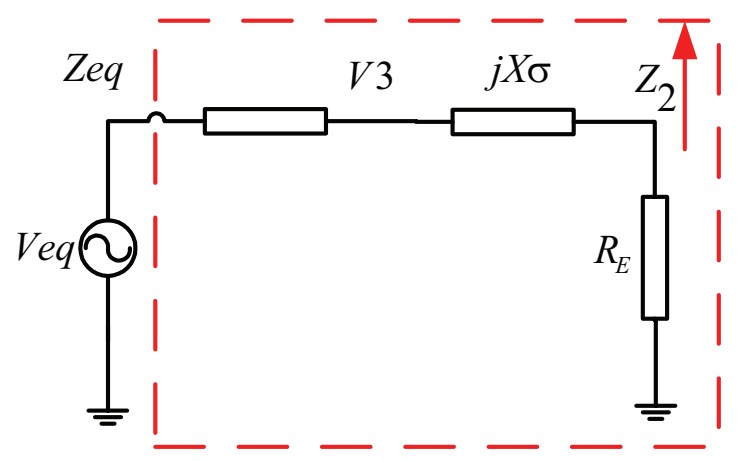

Figure 8. Voltage parameters.

Table 1. Power system parameters

\begin{tabular}{lcccccc}
\hline$V_{1}(\mathbf{p} . \mathbf{u})$ & $X_{\mathrm{L}}^{(1)}(\mathbf{p} \cdot \mathbf{u})$ & $X_{\sigma}(\mathbf{p} \cdot \mathbf{u})$ & $R_{\mathrm{s}}+j X_{\mathrm{s}}(\mathbf{p} . \mathbf{u})$ & $T_{\mathrm{E}}(\mathbf{p} \cdot \mathbf{u})$ & $T_{\mathrm{n}}(\mathbf{p} \cdot \mathbf{u})$ \\
\hline 1.1 & 0.15 & 0.05 & 1.5 & 1.0 & 0.1 & 30 \\
\hline
\end{tabular}

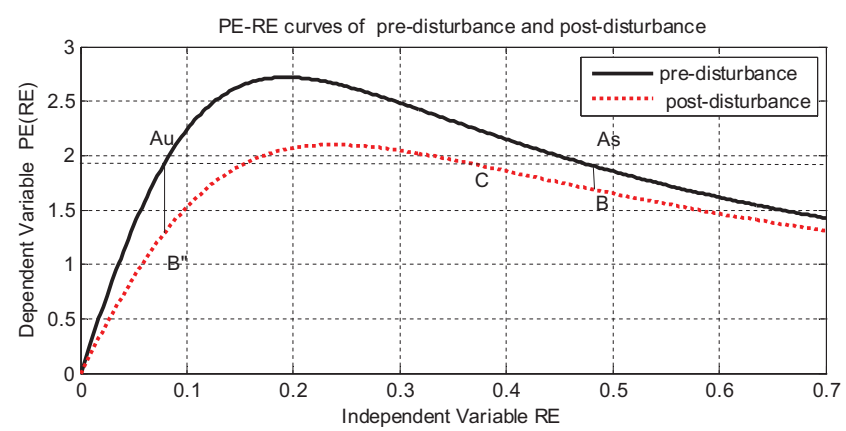

Figure 9. $\mathrm{V}_{3-\mathrm{n}}$ curves of pre-disturbance and postdisturbance.

According to equation (35), since $V_{3}(n)<V_{30}$, then the ratio $n$ will increase. According to the convex characteristic of function $V_{3}(n)$, when $n<n_{\text {cr }} V_{3}(n)$ increases while $n$ increases. In the end, the system reaches point $C$, and therefore, $A^{S}$ is a stable equilibrium point. On the other hand, if the system initially operates at point $A^{u}$ on curve1 (pre-disturbance), the system will jump to point $B^{1}$ on curve2 (post-disturbance) for the same disturbance. According to equation (35), since $V_{3}(n)<V_{30}$, then the ratio $n$ will increase. Considering the convex characteristic of the function $V_{3}(n)$ for $n>n_{\mathrm{cr}} V_{3}(n)$ decreases with the increase of $n$, thus the ratio $n$ will increase and reach to its upper limit. Therefore $A^{u}$ is an unstable equilibrium point. This is called the reverse action of the OLTC of the continuous model as pointed out in [16]. In Figure 10, the corresponding ratios $n^{s}$ and $n^{u}$ for the equilibrium points $A^{S}$ and $A^{u}$ respectively, have the relationship of $n^{s}<n^{u}$.

In equation (37), let $\partial V_{3} / \partial n=0$, then $V_{3}$ reaches to its maximum i.e., $V_{3, \max }$,for:

$$
n=\sqrt{\frac{Z_{3}}{X_{L}}}
$$

$V_{3, \max }$ depends on the parameters $R_{E}, R_{S}, X_{S}, X_{E}$, and $X_{\sigma}$. It is worthwhile to point out that when equation (41), is satisfied, the system operates at the maximum point of the $P-V$ curve of bus 3. For parameters listed in table (1), $P_{\mathrm{E}}-R_{E}$ curves for pre-disturbance (curve 1 ) and post-disturbance (curve 2) cases are given in Figure 10. This figure shows $P_{\mathrm{E}}-R_{E}$ curves are also convex in 2-diniensional space $\left(P_{\mathrm{E}}, R_{\mathrm{E}}\right) . A^{S}$ is a stable equilibrium point, whereas $A^{u}$ is an unstable one. As shown in this figure, the corresponding dynamic resistances $R_{E}^{S}$ and $R_{E}^{u}$ for equilibrium points $A^{S}$ and $A^{u}$, respectively, have the relationship of $R_{E}^{S}>R_{E^{\prime}}^{u}$ which is quite different from that of $n^{S}$ and $n^{\mathrm{u}}$ in Figure 9.

\subsection{Small Signal Analysis}

In this paper, the small-signal analysis is used to study the voltage stability and determine a small-disturbance voltage stability region for a system. The dynamic model of this system is shown in Figure 6 and described by equations (35) and (36). Suppose that the load-flow equation of the system is:

$$
g(X, Y)=0
$$

where, $\bar{X}=\left[n, R_{E}\right]^{T}$ is the state-variable vector, $\bar{Y}$ is the input/output parameter vector including variables such as voltage $V$, active and reactive power $(P$ and $Q)$ etc. After linearizing the differential equations (35) and (36) and the load flow equation (42) at the equilibrium point, using state variables, the state-space model can be written as follows:

$$
\frac{d \Delta \bar{X}}{d t}=A \Delta \bar{X}=\left[\begin{array}{ll}
a_{11} & a_{12} \\
a_{21} & a_{22}
\end{array}\right]\left[\begin{array}{c}
\Delta n \\
\Delta R_{E}
\end{array}\right]
$$




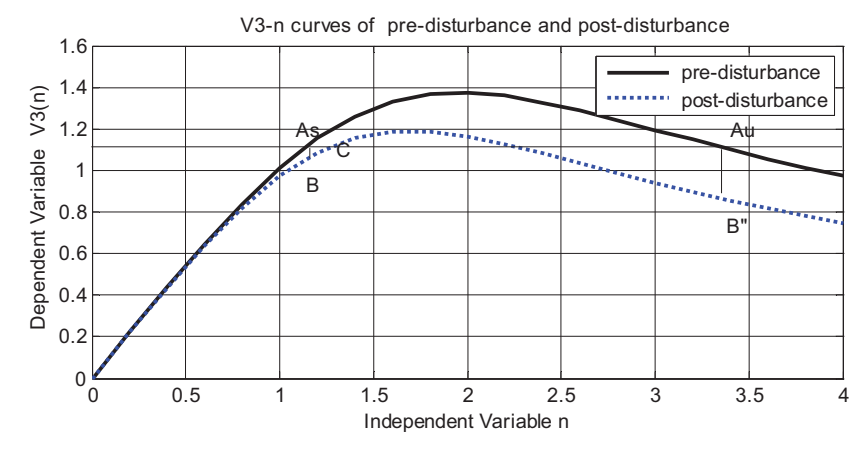

Figure 10. PE-RE curves for pre-disturbance and postdisturbance cases.

The stability criteria of Eqn. 45 are:

$$
\begin{aligned}
& -\left(a_{11}+a_{22}\right)>0 \\
& a_{11} a_{22}-a_{12} a_{21}>0 \\
& U=\left\{\left(n, R_{E}\right) \mid-\left(a_{11}+a_{22}\right)>0, a_{11} a_{22}-a_{12} a_{21}>0\right\}
\end{aligned}
$$

where, $U$ is defined as the small-disturbance voltage stability region. For the system shown in Figure 6, after linearizing the differential equations (35) and (36), and the load flow equations (37)-(40) at the equilibrium point, and eliminating the non-state variables $\Delta P_{\mathrm{E}}$ and $\Delta V_{3}$, the state-space model is transformed into equation (45), where its coefficients are:

$$
\begin{aligned}
& a_{11}=\frac{V_{1} Z_{3}}{T_{n} Z_{1}^{3}}\left(Z_{3}^{2}-n^{4} X_{L}^{2}\right) \\
& a_{12}=\frac{V_{e q}}{T_{n} Z_{E} Z_{2}^{3}}\left[\left(R_{E}+R_{e q}\right) Z_{E}^{2}-R_{E} Z_{2}^{2}\right] \\
& a_{21}=\frac{2 n Z_{3}^{2} V_{1}^{2} R_{E}^{3}}{T_{E} Z_{1}^{4} Z_{E}^{2}}\left(Z_{3}^{2}-n^{4} X_{L}^{2}\right) \\
& a_{22}=\frac{R_{E}^{2} V_{e q}^{2}}{T_{E} Z_{2}^{4}}\left(Z_{5}^{2}-R_{5}^{2}\right)
\end{aligned}
$$

The state space of $\left(n, R_{E}\right)$ in the first quadrant can be partitioned into four regions by curves indicating $a_{11}=0$ and $a_{22}=0$ for equilibrium points in each or the 4 regions $\left(U_{1}\right.$, $U_{2}, U_{3}$ and $U_{4}$ ) the signs of the coefficients in equation (43) and the criteria of stability are derived from the stated expressions, as listed in Table (2).

\section{Conclusion}

This paper discusses the effect of OLTC on the voltage stability via the calculation of the maximum power
Table 2. Signs of coefficients of linearised system and criteria of stability

\begin{tabular}{lcccccccc}
\hline Regions & \multicolumn{3}{c}{ Signs of coefficients } & & \multicolumn{3}{c}{ Signs of criteria } \\
\cline { 2 - 4 } \cline { 6 - 7 } & $a_{11}$ & $a_{12}$ & $a_{21}$ & $a_{22}$ & & $-\left(a_{11}+a_{22}\right)$ & $\left(a_{11} a_{22}-a_{12} a_{21}\right)$ \\
\hline$U_{1}$ & - & - & + & - & & + & + \\
$U_{2}$ & - & - & + & + & uncertain & uncertain \\
$U_{3}$ & + & - & - & - & uncertain & - \\
$U_{4}$ & + & - & - & + & & - & uncertain \\
\hline
\end{tabular}

transmission. It is shown that when the ratio of OLTC increases the maximum reactive power simultaneously increases and the possibility of voltage collapse will decrease under the same load condition. Finally, noticing that the voltage instability is directly affected by load characteristics. Also analyzed the voltage stability of power system with OLTC and dynamic loads, using the state space conception to analyze voltage collapse. The main conclusions of this study are- ; First, the dynamics of both the OLTC and the load can significantly influence the voltage stability of the system. After a small disturbance, whether mode of voltage instability, Past instability or longer-term instability will occur depends on the composition of the load as well as the value of the system reactance. Secondly, the limitation of generator excitation influences the voltage stability by changing the small-disturbance voltage stability region and equilibrium points of the system. When the generator excitation current reaches its maximum, the equilibrium points may disappear, resulting in the system's voltage instability.

\section{References}

1. Taylor C W (1994). Power System Voltage Stability, Appendices:Voltage stability and the power flow problem, McGraw-Hill Ryerson, Limited, Technology \& Engineering, 200-232.

2. Vaithianathan V R, Heinz S C et al. (1995). Dynamics of large constrainted nonlinear systems-a taxonomy theory, Proceedings of the IEEE, vol 83(11), 1530-1561.

3. Ohtsuki H, Yokoyama A et al. (1990). Reverse action of onload tap changer in association with voltage collapse, IEEE Transactions on Power Systems, vol 6(1), 300-306.

4. Liu C, and Vu K T (1989). Analysis of tap-Changer dynamics and construction of voltage stability regions, IEEE Transactions on circuits and systems, vol 36(4), 575-590.

5. Zhu T X, Tso S K et al. (2000). An investigation into the OLTC effects on voltage collapse, IEEE Transactions Power Systems, vol 15(2), 515-521. 
6. Kang P, and Birtwhistle D (2001). Condition monitoring of power transformer on-load tap-changers, Part 2: Detection of ageing from vibration signatures, IEE Proceedings - Generation Transmission and Distribution, vol 148(4), 307-311.

7. Hong Y-Y and Yang Y-L (1998). Evaluation of control sequence for improving voltage stability in power systems, Electric Power Systems Research, vol 47(3), 205-213.

8. Rivas E, Burgos J C et al. (2009). Condition assessment of power OLTC by vibration analysis using wavelet transform, IEEE Transactions on Power Delivery, vol 24(2), 687-694.

9. Rivas E, Burgos J C et al. (2010). Vibration analysis using envelope wavelet for detecting faults in the OLTC tap selector, IEEE Transactions on Power Delivery, vol 25(3), 1629-1636.

10. Liu C C, and Vu K T (1989). Analysis of tap-changer dynamics and construction of voltage stability regions, IEEE Transactions on Circuits and Systems, vol 36(4), 575-590.
11. Vu K T, and Liu C C (1992). Shrinking stability regions and voltage collapse in power systems, IEEE Transactions on Circuits and Systems, vol 39(4), 271-289.

12. Vu K T, and Liu C C (1990). Dynamic mechanisms of voltage collapse, Systems and Control Letters, vol 15, 329-338.

13. Vancutsem T (1988). Dynamic and static aspects of voltage collapse, Proc. Engineering Foundation Conf. on Bulk Power System Voltage Phenomena: Voltage Security and Stability, Potosi (USA), 6.55-6.79.

14. Ohtsuki H, Yokoyama A et al. (1991). Reverse action or onload tap changer in association with voltage collapse, IEEE Transactions on Power Systems, vol 6(1), 300-306.

15. Hill D J (1993). Nonlinear dynamic load models with recovery for voltage stability studies, IEEE Transactions on Power System, vol 8(1), 166-176.

16. Xu W, and Mansour Y (1994). Voltage stability analysis using generic dynamic load models, IEEE Transactions on Power System, vol 9(1), 479-493. 